\title{
Étude des niveaux d'irradiation à la périphérie du centre de Frascati par rapport à la radioactivité naturelle $\left(^{*}\right)$
}

\author{
F. LUCCI, M. PELLICCIONI $(* *)$
}

(Manuscrit reçu le 17 septembre 1975)

\begin{abstract}
RÉSUMÉ
On présente les résultats des contrôles dosimétriques effectués à la périphérie du Centre de Frascati à l'aide de dosimètres thermoluminescents, à partir de 1969.

L'exposition annuelle moyenne, mesurée pendant toute la période 1969-1974, se situe à $206 \mathrm{mR} / \mathrm{an}$, tandis qu'on ne peut remarquer aucune tendance significative. En particulier, on ne note ni l'influence de l'arrêt des machines, ni d'effets saisonniers.

Les mesures effectuées à la périphérie sont également comparées avec quelques mesures de la radioactivité naturelle effectuées dans la région de Frascati et à Rome. On peut ainsi estimer que la contribution des machines à la dose totale à la périphérie du Centre n'est qu'une petite fraction de la radioactivité naturelle et de la limite maximale admissible pour la population dans son ensemble.
\end{abstract}

\begin{abstract}
The results of dosimetric controls carried out with thermoluminescent dosimeters along the boundaries of the Frascati Center since 1969 are presented. The average yearly exposure observed during the period 1969-1974 is $206 \mathrm{mR} / \mathrm{y}$, while no significant tendency is evident. In particular neither effects of accelerator shutdowns nor seasonal ones are noticed.

The measurements at the boundary are also compared with measurements of natural background carried out near Frascati and in Rome. Thus we can estimate that the machine contribution to the total dose along the boundaries is equal to a small fraction of either the natural background or the general population dose limit.
\end{abstract}

(*) Communication présentée au « 3rd European IRPA Congress ", Amsterdam, 13-16 mai 1975.

(**) CNEN, Laboratori Nazionali di Frascati. Casella postale 70, 00044 Frascati (Roma) Italie.

RADIOPROTECTION, VOL. $10-\mathrm{N}^{\circ} 3$ 


\section{INTRODUCTION}

Normalement, les projets d'installations nucléaires ou de machines radiogènes de grande puissance, telles que les accélérateurs de haute énergie, doivent tenir compte du problème de la limitation des doses, de manière à garantir, sur les lieux de travail et aux alentours, des niveaux d'irradiation inférieurs aux doses maximales admissibles pour les diverses catégories de personnes exposées.

Par la suite, des contrôles dosimétriques adéquats effectués dans le cadre de la surveillance de physique sanitaire vérifieront que les limites maximales imposées sont bien respectées.

Dans le présent travail, nous nous sommes principalement intéressés aux problèmes de radioprotection dans les zones limitrophes du Centre de Frascati où sont installés des accélérateurs de haute énergie, un électrosynchrotron de $1 \mathrm{GeV}$, un accélérateur linéaire de $400 \mathrm{MeV} 36 \mathrm{~kW}$, un anneau d'accumulation pour électrons et positons de $1,5 \mathrm{GeV}$ et un certain nombre de machines de moindre importance.

Pour la population aux alentours du Centre, nous avons adopté la limite de $170 \mathrm{mrem} / \mathrm{an}$ comme dose moyenne maximale admissible; cette manière de voir n'est pas toujours partagée; sans entrer plus avant dans le sujet, nous faisons observer que l'adoption de la limite supérieure (500 mrem/an) comporterait, pour la loi italienne, la nécessité d'assurer la surveillance d'ambiance dans les zones extérieures au Centre, ce que nous préférons éviter, ne serait-ce que pour des raisons psychologiques évidentes.

La limite choisie a évidemment des répercussions sur l'organisation de la radioprotection, soit sur la conception des dispositifs de protection, écrans en particulier, soit sur la réglementation du fonctionnement des machines, soit encore sur l'appareillage de contrôle qui doit être capable, entre autres, d'évaluer des doses de l'ordre de $170 \mathrm{mrem} / \mathrm{an}$.

En général, dans les divers centres dans lesquels on rencontre ce type de problème, on a recours à des points de contrôle équipés avec des chambres d'ionisation, des détecteurs de neutrons, des dosimètres à intégration, etc. et à des programmes de surveillance périodique réalisés par du personnel spécialisé.

Dans le cas particulier du Centre de Frascati, le dépassement des $170 \mathrm{mrem} / \mathrm{an}$ dans la zone extérieure au Centre est extrêmement improbable si l'on considère les caractéristiques des accélérateurs présents et des systèmes de protection correspondants. De plus, étant donné les dimensions mêmes du Centre, un tel dépassement serait certainement accompagné de dépassements analogues des limites prévues pour les zones accessibles à l'intérieur du Centre. Dans cette dernière hypothèse, interviendraient alors normalement les systèmes de contrôle et de prévention.

Ainsi s'est-on décidé à limiter le contrôle à la périphérie du Centre aux doses gamma mesurées à l'aide de dosimètres thermoluminescents de type commercial. 
En ce qui concerne la composante neutronique, en l'absence de dosimètre intégrateur suffisamment sensible, on préfère recourir à l'extrapolation des données recueillies au voisinage des machines, soit avec des films dosimètres, soit avec des " rem-counters "; cette extrapolation peut être étendue jusqu'aux limites du Centre.

La méthode suivie est évidemment justifiée du fait de la prépondérance de la composante électromagnétique dans les champs de rayonnements autour des accélérateurs d'électrons, du moins pour les énergies de nos machines, spécialement dans les cas d'écrans éventuellement défectueux qui pourraient donner lieu à des fuites de rayonnement de valeurs excessives.

\section{2. ÉVALUATION DES NIVEAUX DE DOSES A LA PÉRIPHÉRIE DU CENTRE DE FRASCATI}

La figure 1 montre la position des deux principales machines du Centre et celle des dosimètres de contrôle placés à la périphérie ainsi que les valeurs moyennes relevées annuellement ces dernières années. La moyenne des valeurs

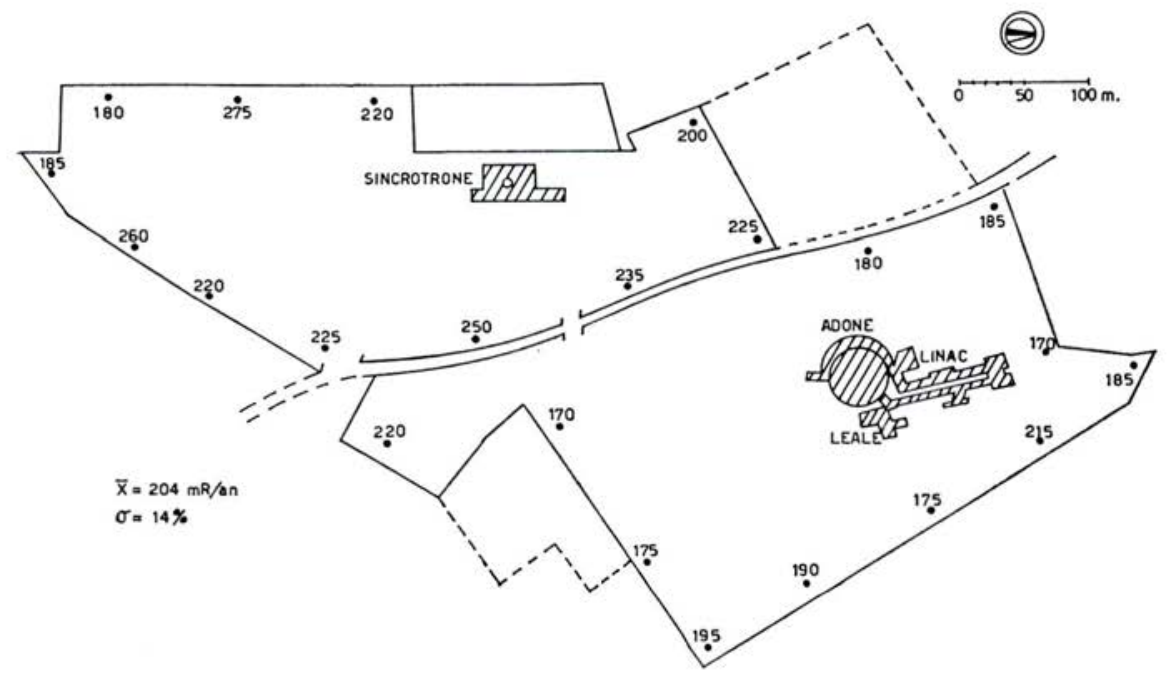

FIG. 1. - Points de contrôle et expositions moyennes relevées au cours de la période 1972-1974.

indiquées est de $204 \mathrm{mR}$, avec une déviation standard de $14 \mathrm{p}$. cent; la demi étendue, par contre, est de 26 p. cent. En examinant l'ensemble des résultats, il convient, de toute façon, de tenir compte des circonstances suivantes :

a) les expositions indiquées comprennent la radioactivité naturelle (d'origine terrestre et cosmique) et le mouvement propre des dosimètres;

b) ces résultats ne correspondent pas toujours à des périodes coïncidant avec les années solaires et ils ont été, par conséquent, normalisés à 365 jours. 
Dans la figure 2, la distribution des débits d'exposition mesurés tous les trois mois dans tous les points contrôlés est comparée, année par année et pour toute la période, avec la distribution de la réponse du système dosi-

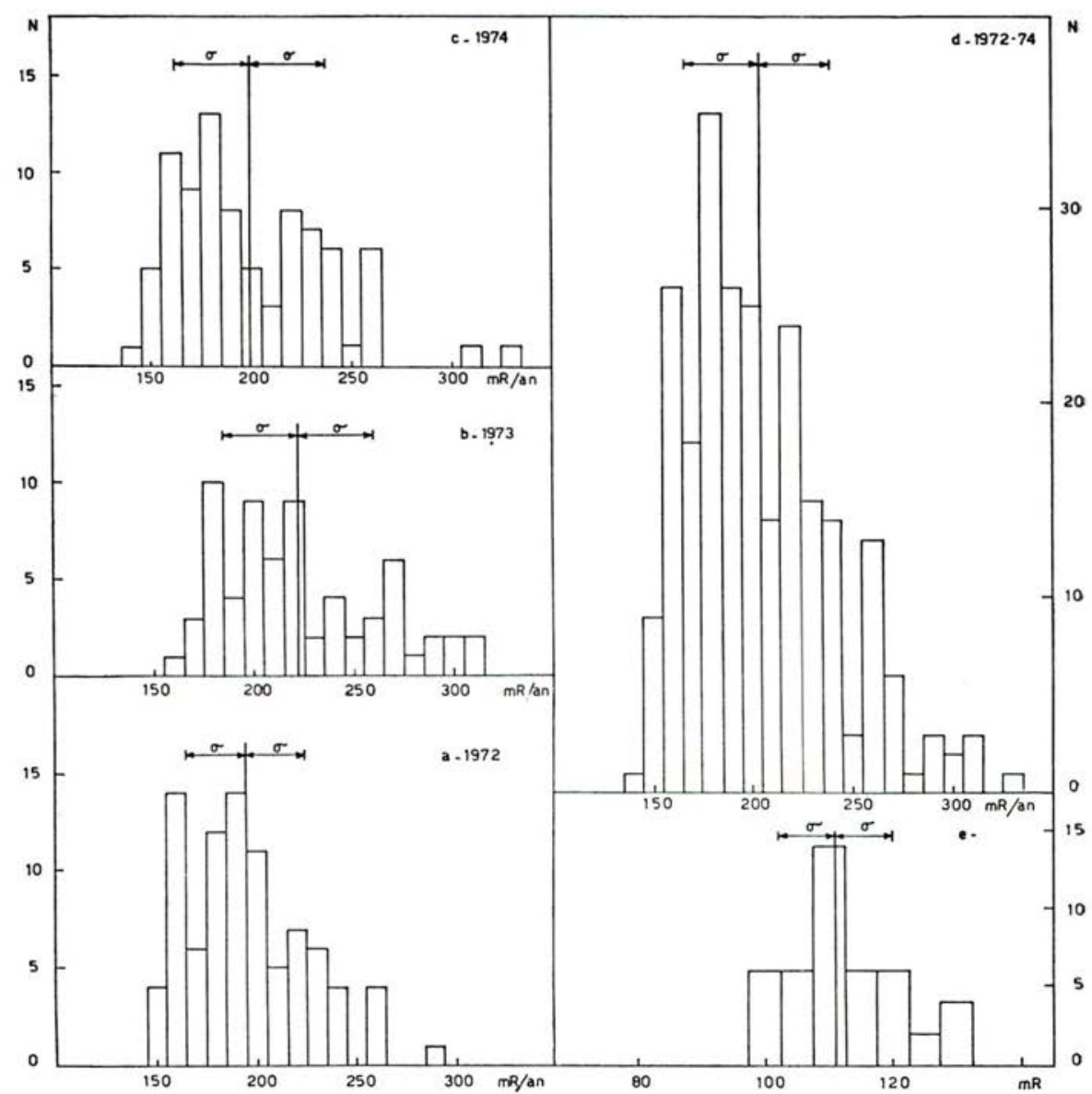

FIG. 2. - Distribution des débits d'exposition mesurés année par année $(a, b, c)$ et durant toute la période 1972-1974 $(d)$ et de la réponse du système de mesure pour une exposition au ${ }^{60} \mathrm{Co}(e)$.

métrique obtenue au cours de l'étalonnage. Dans la même figure, sont aussi indiquées les valeurs moyennes annuelles, au moyen des lignes verticales en trait plein, et les écarts-types. On remarque que d'un écart-type d'environ 6 p. cent pour l'étalonnage on passe, pour les mesures à la périphérie, à $15-20$ p. cent environ. 
Sur la figure 3 on montre la variation au cours du temps des mesures à la périphérie, extrapolées à 365 jours, à partir de 1969, année du début de ce type de contrôle. La droite horizontale représente la moyenne sur toute la période 1969-1974. On ne remarque aucune tendance significative, les petites variations autour de la valeur moyenne de $206 \mathrm{mR} / \mathrm{an}$ pouvant être attribuées à des circonstances fortuites comme, par exemple, des changements même peu importants de la position de quelques dosimètres; en particulier, on ne note même pas d'influence due aux arrêts prolongés des machines, indiqués, eux aussi, sur la figure.

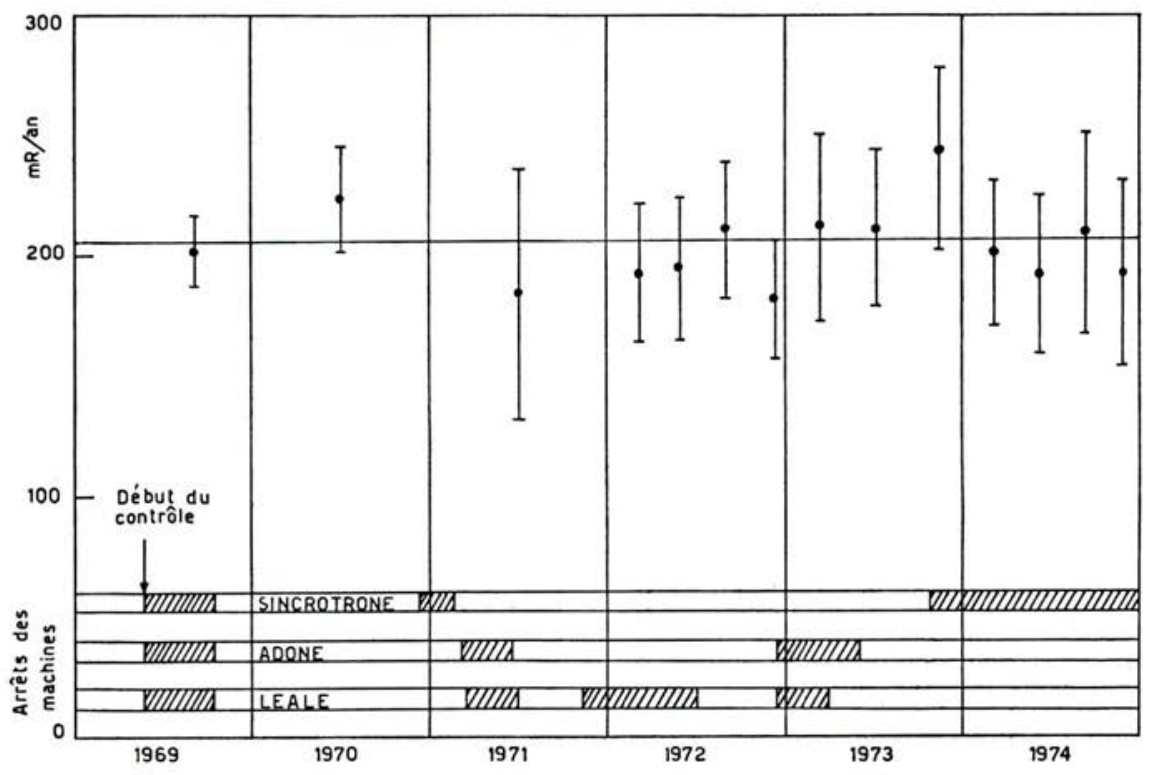

FIG. 3. - Variation, dans le temps, de l'exposition moyenne à la périphérie. La droite horizontale représente la valeur moyenne; les traits verticaux indiquent les écarts-types.

Dans le but de mieux évaluer la part due au fonctionnement des machines, qui semble négligeable étant donné les observations déjà faites, on a jugé utile d'approfondir l'étude du bruit de fond naturel dans les zones limitrophes du Centre. Nous avons considéré le fait que la concentration en U, Th, K, dans les roches et matériaux volcaniques peut atteindre des valeurs relativement élevées, particulièrement dans les zones volcaniques de l'Italie centrale et méridionale $[1,2]$.

Quoique partiellement, on avait, depuis 1969, effectué un certain nombre de mesures, surtout dans les habitations situées à Rome et aux alentours du Centre.

On avait mesuré des valeurs moyennes de $250 \mathrm{mR} / \mathrm{an}$, avec des minimums autour de $110 \mathrm{mR} / \mathrm{an}$ et des maximums de $375 \mathrm{mR} / \mathrm{an}$. Une série de mesures

vOL. $10-\mathrm{N}^{\circ} 3$ 
conduites de façon plus systématique a été effectuée au cours de l'année 1974, en laissant les dosimètres aux points de mesure durant l'année entière et en les lisant tous les trois mois. La moyenne des valeurs trouvées dans des jardins situés à quelques kilomètres de distance du Centre a été de $259 \mathrm{mR} / \mathrm{an}$ avec un écart-type de $15 \mathrm{p}$. cent. Il est superflu de rappeler que ces valeurs comprennent le bruit de fond naturel des rayonnements ainsi que le bruit de fond propre des dosimètres.

En ce qui concerne ce dernier, on l'a mesuré à l'aide de dosimètres conservés dans un petit château de plomb de $10 \mathrm{~cm}$ d'épaisseur, qui ont enregistré environ $0,22 \mathrm{mR} /$ jour, avec un écart-type de $7 \mathrm{p}$. cent. Une partie de cette dose doit être attribuée aux effets de la composante $\mu$ des rayons cosmiques $(0,05 \mathrm{mR} /$ jour $)$ et de la contamination radioactive naturelle du plomb. Puisque une concentration de $10^{-12} \mathrm{Ci} / \mathrm{g}$ de ${ }^{226} \mathrm{Ra}$ dans le plomb produirait une intensité d'exposition de presque $0,05 \mathrm{mR} / \mathrm{jour}$, nous pouvons estimer que le bruit de fond propre des dosimètres ne dépasse pas 0,10 à $0,15 \mathrm{mR} /$ jour.

A la lumière de ce qui précède, on peut donc affirmer que la contribution des machines du Centre aux doses gamma mesurées à la périphérie est tellement modeste qu'il est difficile de la distinguer du bruit de fond naturel et n'est, de toute façon, qu'une petite fraction de la dose moyenne maximale admissible de $170 \mathrm{mrem} / \mathrm{an}$ fixée pour les zones en dehors des zones surveillées.

En ce qui concerne la composante neutronique, à cause de l'imprécision des films dosimètres, en particulier pour les neutrons d'énergie inférieure à $2 \mathrm{MeV}$, nous avons considéré spécialement les mesures effectuées avec les "rem-counters ». Pour l'extrapolation des données, on a tenu comptedes lois empiriques de l'effet de ciel obtenues en son temps pour les machines et avec la géométrie du Centre de Frascati [3, 4].

En introduisant toute une série d'hypothèses restrictives dans l'analyse et dans l'élaboration des données, que nous ne pouvons pas rappeler ici, on a pu établir pour la contribution de la composante neutronique, une limite supérieure qui est, tout au plus, de l'ordre de 1 à 2/10 de la dose limite pour la population.

De ce que l'on vient d'exposer, on peut donc affirmer que les doses à la périphérie du Centre de Frascati représentent seulement une petite fraction de la dose limite pour la population. On peut, en outre, estimer que, compte tenu de la distance du Centre aux habitations voisines, de l'atténuation dans les murs, etc., les doses effectivement reçues par les habitants les plus proches sont sûrement inférieures à quelques centièmes de la limite de dose.

\section{INDICATIONS SUR QUELQUES RÉSULTATS DES MESURES EFFECTUÉES DANS DES HABITATIONS}

Nous estimons qu'il est intéressant de rapporter ici quelques résultats de mesures faites au cours de l'année 1974 à l'intérieur des habitations situées à Rome et aux alentours du Centre [5]. Ces mesures ont été conduites simultanément avec celles précédemment citées en considérant les zones extérieures 
aux habitations (jardins, etc.). La distribution des expositions annuelles enregistrées à l'intérieur est montrée sur la figure 4 . La valeur moyenne est de $304 \mathrm{mR} / \mathrm{an}$ avec un écart-type de 19 p. cent. On n'a pas observé de variations saisonnières appréciables.

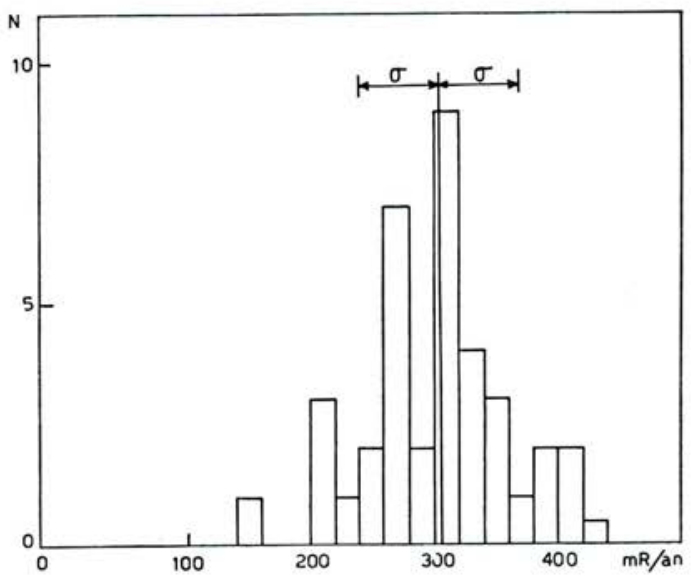

FIG. 4. - Distribution des expositions annuelles enregistrées à l'intérieur des habitations.

Une autre preuve réside dans la mesure de l'exposition de personnes sûrement non exposées à des sources artificielles de rayonnements. Nous avons distribué à sept personnes des dosimètres thermoluminescents lus trimestriellement [5]. Les expositions annuelles, dans ce cas, sont comprises entre 180 et $320 \mathrm{mR} / \mathrm{an}$ avec une moyenne de $249 \mathrm{mR} / \mathrm{an}$ et un écarttype de $17 \mathrm{p}$. cent.

Il semble donc raisonnable d'estimer à $200-250 \mathrm{mR} / \mathrm{an}$, non compris le bruit de fond des dosimètres, les irradiations d'origine naturelle auxquelles sont exposés des individus ayant des modes de vie et des habitudes comparables à ceux des familles des employés du Centre de Frascati.

\section{CONCLUSION}

Comme on l'a vu, la contribution des machines au niveau d'irradiation à la périphérie du Centre de Frascati est sûrement inférieure à quelques dixièmes de la limite de dose pour la population. La dose effectivement reçue par les personnes les plus proches du Centre a été, à son tour, estimée inférieure à quelques centièmes de la limite de dose.

La difficulté de mesure de doses aussi petites, liée au fait que des doses comparables et souvent supérieures dues à la radioactivité naturelle sont fréquemment reçues dans la vie quotidienne, nous laissent assez perplexes 
quant au projet, plusieurs fois présenté, d'une réduction des limites de dose et quant à ses conséquences sur le travail pratique de radioprotection.

Dans une telle éventualité, il serait évidemment très problématique d'établir des contrôles expérimentaux capables d'évaluer d'une manière significative des quantités la plupart du temps équivalentes aux fluctuations de la radioactivité ambiante. Ceci est, de fait, confirmé par l'expérience pratique de quelques Centres, comme celui de Stanford, où l'on cherche à mesurer des niveaux de l'ordre de $1 / 100$ de la limite de dose (1,7 mrem/an). Ceux qui doivent effectuer les mesures les reconnaissent volontiers comme privées de signification expérimentale [6].

Il serait donc nécessaire de remplacer les contrôles par des évaluations théoriques et par des extrapolations de mesures effectuées en des points plus voisins des sources.

Nous n'entendons pas affirmer que la dite réduction ne doit pas être opérée, cela ne serait ni de notre compétence, ni de notre ressort. Nous tenons plutôt à rappeler la signification statistique de la limitation des doses aux populations. Il n'y a, en effet, aucun doute que la « dose maximale admissible significative du point de vue génétique » pour la population dans son ensemble soit un concept purement statistique et non susceptible de mesure. C'est pourquoi nous pensons qu'une réduction des limites de dose ne doit et ne peut pas comporter la nécessité de mesures directes de valeurs inférieures aux nouvelles limites.

Sur le problème même de l'opportunité d'une réduction, nous nous limitons à observer que, par cohérence, il faudrait considérer aussi la possibilité d'introduire des limitations allant jusqu'à la liberté personnelle, par exemple, quant au choix d'un lieu d'habitation ou des matériaux de construction des maisons [7]. Il serait, en outre, souhaitable que le problème de la réduction des doses dues à la pratique médicale soit affronté avec une attention plus grande qu'actuellement.

Enfin, il nous plaît de conclure en observant que, déjà actuellement, les physiciens sanitaires travaillent pour minimiser les doses effectivement reçues par les populations. Cela en observant les principes formulés par la CIPR [8, 9] sur la nécessité d'éviter toutes les expositions inutiles et de maintenir raisonnablement aussi basses que possible celles retenues nécessaires.

\section{BIBLIOGRAPHIE}

[1] Imbo G., Gasparini P., Luongo et G. Rapolla A. Contributions to the volcanological researches by determination of the radioactivity of eruptive products, Bull. Volc., 1968, 32, 2-25.

[2] Civetta L. et Gasparini P. A review of $U$ and Th distributions in recent volcanoes from southern Italy: magnetological and geophysical implications, Osservatorio Vesuviano, Ercolano, 1972.

[3] Ladu M., Pelliccioni M., Picchi P. et Verri G. A contribution to the skyshine study, Nucl. Instr. Meth., 1968, 62, 51. 
[4] LuCci F. et Pelliccioni M. Esperienza di sorveglianza fisica delle radiazioni ai confini del Centro di Frascati, Frascati Report, LNF-74/38 (R), 1974.

[5] Lucci F., Paganelli M. et Pelliccioni M. Risultati di un'indagine campione sul fondo naturale di radiazioni nella zona di Roma, LNF-75/24 (R), 1975.

[6] Jenkins T. M. Accelerator boundary doses and skyshine, Health Physics, 1974, 24, 251.

[7] JАСовI W. Beziehungen zwischen der Strahlendosis und dem somatischen Strahlenrisiko, Atomwirtschaft, 1974, 278.

[8] Recommendations of the International Commission on Radiological Protection (Adopted September 17, 1965), ICRP Publication 9. Oxford, Pergamon press, 1966.

[9] Implications of the Commission's Recommendations that doses be kept as low as readily achievable, ICRP Publication 22, Oxford, Pergamon press, 1973. 